\title{
Modeling the glycolysis dynamics
}

\author{
A. Kotipalli ${ }^{1}$, C.K. Mitra ${ }^{2 *}$ \\ ${ }^{1}$ Centre for Development of Advanced Computing, Pune, India \\ ${ }^{2}$ University of Hyderabad, Hyderabad, India \\ *e-mail:c_mitra@yahoo.com
}

Key words: metabolic dynamics, kinetics model, reaction pathway, systems biology, glycolysis

Motivation and Aim: Glucose concentration must be regulated relatively tightly within the cell. In the glycolytic pathway, the sequence of biochemical reactions is regulated at several places: early at hexokinase, somewhere in the middle during the conversion of fructose-6-phosphate to fructose bis-phosphate by phosphofructose kinase and in the late stages the concentration of pyruvate is regulated by pyruvate kinase. Although studied as a stand-alone pathway, glycolysis is tightly coupled with the TCA cycle, the pentose phosphate pathway and the glycogen metabolism pathway. Different cells have their own metabolic profiles and several cell types are also under external hormonal controls. Methods and Algorithms: In this study we have used a simulation (based on Octave) and used an extension of Michealis-Menten equation for the reaction kinetics. We also used an (empirical) extension of the Quasi-Steady-State (QSS) approximation to reduce the effective number of parameters. In the current simulation, we have not considered the coupling with other networks nor the effect of the gene regulation on glycolysis. We have kept the enzyme concentrations (molar numbers) equal for all the enzymes in the pathway. The substrate concentrations were often expressed in terms of the relevant $\mathrm{K}_{\mathrm{M}}$ (the dissociation constant of the enzyme substrate complex) to reduce the apparent complexity. The final set of differential equations were solved by Octave $[1,2]$.

Results: The results of the present simulations are generally in line with expected results and we do not expect an accurate match with the cellular level concentrations of the different metabolites.

Conclusion: Although the methodology uses approximations and the quantitative effects of the approximations have not been studied here, we believe the tools presented in this study are effective in general understanding of the roles played by different regulators. Additional cellular level (genetic level) or external induced (hormonal effects) have not been considered.

Acknowledgements: Financial Support from the University Grants Commission (https:// www.ugc.ac.in) to one of the authors (CKM) is gratefully acknowledged (2015-2017).

\section{References}

1. Korla K., Mitra C.K. (2014) Modeling the Krebs cycle and oxidative phosphorylation. J. Biomol. Struct. Dyn. 32(2):242-256.

2. Korla K., Mitra C.K. (2014) Kinetic modeling of coupled transport across biological membranes. Ind. J. Biochem. Biophys. 51(2):93-99. 